\title{
Medication Injection Safety Knowledge and Practices among Health Service Providers in Korea
}

\author{
Hyeong-Il Lee ${ }^{1}$, Ji-Eun Choi ${ }^{1}$, Sol-Ji Choi ${ }^{1}$, Eun-Bi Ko² \\ ${ }^{1}$ National Evidence-based Healthcare Collaborating Agency, Seoul, Republic of Korea \\ 2 Yonsei University Graduate School, Wonju, Republic of Korea
}

Purpose: Outbreaks resulting from medication injections have recently been on the rise in Korea despite various established guidelines. The objective of this study was to assess the degree to which healthcare professionals are aware of safe injection practice guidelines and to account for the adherence to and the deviation from safe injection guidelines formulated by healthcare providers.

Methods: In November 2016, a cross-sectional anonymous questionnaire covering general characteristics of injections, patient safety culture, awareness of safe injection practices, and adherence to and barriers to safe injection guidelines was issued to healthcare providers who administer medication injections or manage and supervise these injections ( $N=550$ ). Multivariate logistic regression analysis via enter method was performed to define the influencing factors of adherence of safe injection practices.

Results: On average, respondents adhere to 17 of the 24 guidelines. Multivariate logistic regression found that those who were more likely to adhere to safe injection guidelines either underwent a patient safety training experience within the last year, provided care in a setting characterized by a highly developed patient safety culture, or were employed as physicians or nurses, as opposed to some other type of care provider. Barriers to safe injection guidelines were attributable to; thoughts of waste to discard leftover medicine, provisions that made adherence cumbersome, a weak culture of compliance, and insufficient amounts of injectable medicine, products, and education.

Conclusions: The results of this study indicate that controllable factors like training experience of healthcare providers and patient safety culture were positively associated with adherence to safe injection practices. It was suggested that the training of healthcare providers on safe injection practices be a continuous process to promote patient safety. Additionally, there should be an increased focus on developing and implementing policies to improve patient safety culture from a prevention rather than post-management perspective.

Key words: Injections, Safety management, Guideline adherence

Received: May.10.2019 Revised: May.30.2019 Accepted: Jun.07.2019

Correspondence: Ji-Eun Choi

04554, Namsan Square 7F, 173 Toegye-ro, Jung-gu, Seoul, Korea

Tel: +82-2-2174-2880 E-mail: jechoidneca.re.kr

Funding: None Conflict of Interest: None

Quality Improvement in Health Care vol.25 no.1

(C) The Author 2019. Published by Korean Society for Quality in Health Care; all rights reserved

52 Quality Improvement in Health Care 


\section{Introduction}

Over the last two decades, outbreaks and patient concerns about unsafe injection practices reports have been on the rise even in developed countries [1-5]. Though a total of 61 healthcare-associated hepatitis B and C outbreaks were recorded by the Centers for Disease Control and Prevention during 2008 2017 in the U.S., the numbers reported here may have been greatly underestimated because of the long incubation period (up to 6 months) and typically asymptomatic course of acute hepatitis $B$ and C infections [6]. In January 2005, a few malignant cases of recurrent subcutaneous abscesses in the gluteal region were reported in Icheon City [7]. In October 2012, 27 patients were hospitalized due to septic arthritis caused by a presumed NTM infection, after having been given triamcinolone injections at a single clinic [8].

Medication injection-associated infection can be prevented when healthcare providers adhere to infection control guidelines [9]. However, despite various established guidelines, including the Association for Professionals in Infection Control and Epidemiology position paper [10] and World Health Organization [11], unsafe practices have continued, including use of a single dose vial for more than one patient, reuse of syringes [12], and leaving a needle or spike device for later reuse in the same medication vial [13]. It is therefore important to assess the awareness, practices, and adherence of service providers and identify the barriers to adherence to infection control guidelines, to promote patient safety in medication injections. The three common barriers to dedicating medication vials to individual patients are medication shortages, a desire to reduce medication waste, and the high cost of medications [13]. The barriers to safe injection practice could be affected by economic status, social aspects, or organizational patient safety culture [14-15].

Recently, there was a report on the reuse of needles and syringes in conjunction with an increase in cases of HCV at a clinic in Korea [16]. Thus, the objective of this study was to assess the awareness of safe injection practices of service providers, and the adherence and barriers to guidelines of safe injection practice in various settings to promote injection safety.

\section{Methods}

\section{Survey subjects and methods}

A cross sectional study using a structured questionnaire survey was conducted from November 24 to 28, 2016, to understand the level of knowledge, adherence, and barriers to safe injection practice of health service providers. In consideration of the convenience of respondents, both online and offline methods were used to collect data. The respondents were 1,000 professionals providing healthcare at various clinical settings, including members of the Korean Academy of Family Medicine, Korean Nurses Association, Korean Licensed Practical Nurses Association, and Korean Society for Quality in Health Care. They were asked if they were working on medication injection or were managing and supervising medication injections the last one year to screen respondents. Out of the 1,000 respondents, the data of 550 respondents 
were analyzed after excluding 450 respondents whose responses were "No" to both the screening questions.

The questionnaire covered the following five areas: 1) general characteristics, 2) awareness of safe injection practice, 3) adherence to guidelines of injection practice, 4) barriers to adhering to the guidelines of injection practice, and 5) patient safety culture. The questions regarding awareness of safe injection practice, adherence, and barriers were developed through literature reviews and in consultation with experts involved with patient safety. The questions went through a pilot test and thereafter revised before this study was carried out. Patient safety culture was assessed using the Korean version of the self-reported Agency for Healthcare Research and Quality (AHRQ) patient safety questionnaire measured on a 5-point Likert scale [17]. IRB approval from the National Evidence-Based Healthcare Collaborating Agency (IRB No. NECAIRB16-004) was obtained before carrying out this study.

\section{Measurement}

1) Awareness of safe injection practice

Awareness of safe injection practice was assessed with 12 questions to which the survey participants responded "Yes" or "No." Each question with the answer "yes"-received one point, and the total score ranged from 0 to 12 points.

2) Adherence to the guidelines of safe injection practice

24 questions were used to assess adherence to the guidelines regarding medication injection [9-
13]. This included practices related to injections, syringes, and injection needles for the last one year. Items were measured on a 5-point Likert scale (never, rarely, occasionally, frequently, very frequently) (Cronbach's $\alpha=$.784).

3) Barriers to the adherence of guidelines of safe injection practice

Barriers to the adherence of guidelines of safe injection practice were identified using 14 questions to which the survey participants responded "Yes" or "No", developed by researchers based on a literature review. Respondents were asked if they agreed to the barriers mentioned (Cronbach's $\alpha=$.786).

\section{4) Patient safety culture}

Validated Korean version of "Hospital Survey on Patient Safety Culture" (HSPSC) questionnaire developed by Agency for Healthcare Research and Quality (AHRQ) was used to assess the patient safety culture [17]. The HSPSC consists of 44 questions that are divided into the following sections: A. Work environment of each department (18 questions). B. Immediate superior/manager (4 questions). C. Communication and procedures (6 questions). D. Frequency of reported accidents (3 questions). E. Hospital (11 questions). F. Patients' safety level (1 question). G. Number of accidents related to patient safety reported (1 question). The sections A through E were assessed on a 5-point scale of "Not at all," "No," “Average," "Yes," or "Absolutely yes" for each question while sections $F$ and $G$, which concern the safety culture score of patients, were assessed on scores ranging from 42 to 210 points (Cronbach's $\alpha$ $=.782)$. 


\section{5) General characteristics}

General characteristics were assessed using 12 questions regarding the medical institution type, type of occupation, work experience, status of participation in training held by the institution for the past one year, status of participation in training held by an external institution for the past one year, and status of possession of injection safety-related guidelines/regulations in the working institution.

\section{Statistical analysis}

The data collected in this study were presented as frequencies, means, and standard deviations. To identify potential factors influencing adherence to safe injection practices, the variables of general characteristics, awareness of safe injection practices, and patient safety culture were dichotomized by the mean to facilitate interpretation of data, and preliminary univariate analyses were conducted using chi-square or ANOVA $(p<.05)$. Then, the final determinants of adherence to safe injection practice guidelines were identified using multivariate logistic regression analysis via enter variable selection method. The reliability of questionnaire was verified using Cronbach's $\alpha$. The significance level was set at $p<.05$ (two-tailed), and all data analyses were conducted using SPSS 19.0 (Chicago, IL) for Windows.

\section{Results}

\section{General characteristics of respondents and level of patient safety culture}

Table 1 shows the general characteristics and patient safety culture of respondents. Of the respondents, 303 (55.1\%) were working for a tertiary or general hospital, 66 (12.0\%) were physicians, 258 (46.9\%) were nurses, and 258 (41.1\%) were nursing assistants. $51.1 \%$ of the respondents had been working for less than five years. 355 (64.5\%) of the respondents had training experience during the last one year. The mean score of patient safety culture was $144.47 \pm 19.4$.

Table 1. General characteristics and level of safety culture of patients

\begin{tabular}{|c|c|c|}
\hline & Classification & $\mathrm{n}(\%)^{1)}$ \\
\hline \multirow[t]{2}{*}{ Institution type } & Tertiary or general hospital & $303(55.1)$ \\
\hline & Clinics, specialized hospital, nursing home, public health center & $247(44.9)$ \\
\hline \multirow[t]{3}{*}{ Type of occupation } & Physician & $66(12.0)$ \\
\hline & Nurse & 258 (46.9) \\
\hline & Nursing assistants and others & $226(41.1)$ \\
\hline \multirow[t]{2}{*}{ Work experience } & 5 years or less & $281(51.1)$ \\
\hline & More than 5 years & 269 (48.9) \\
\hline \multirow{2}{*}{$\begin{array}{l}\text { Training experiences } \\
\text { (for the last one year) }\end{array}$} & Yes & $355(64.5)$ \\
\hline & No & $191(34.7)$ \\
\hline Awareness of safe injection practice & Mean \pm standard deviation & $9.7 \pm 1.5$ \\
\hline \multirow{2}{*}{$\begin{array}{l}\text { Guidelines/regulations on drug storage and } \\
\text { management }\end{array}$} & Yes & 500 (90.9) \\
\hline & No & $46(8.4)$ \\
\hline Patient safety culture & Mean \pm standard deviation & $144.5 \pm 19.4$ \\
\hline
\end{tabular}

\footnotetext{
1) The total is not $100 \%$ in some cases due to missing variables
} 


\section{Original Articles}

\section{Awareness of safe injection practice}

The mean score for the awareness of safe injection practices was 9.8 out of 12 points (Table 2). $42.7 \%$ of the respondents gave incorrect answers to questions regarding "Disinfecting the injection area is performed for 30 seconds" and "Alcohol-soaked cotton in a container is not prepared in advance". This was the highest number of incorrect answers.

Table 2. Awareness of safe injection practice

$(\mathrm{n}=550)$

\section{Question}

1. In medication injection, only injection needles are contaminated.

2. Contaminated syringes or injection needles can be fully identified by the naked eye.

3. Once injection needles are replaced, syringes can be reused.

If medication must be dissolved for injection, a large amount of solvent fluid or bottle can be used in combination.

5. For skin disinfection prior to injection, the skin is disinfected with an alcohol

6. Disinfecting the injection area is performed for 30 seconds

7. Once the skin surface has dried after skin disinfection, the effect of disinfection disappears.

8. Alcohol-soaked cotton in a container is not prepared in advance

Confirmation is to be made whether multiple patient use is written in the

9. drug manual containing prescription cautions etc., included in the injection package.

10. TPN solution must be discarded 48 hours after opening.

11. You are aware of injection-related infection incidents that occurred in South Korea.

12. You are familiar with injection safety use guidelines published in South Korea. swab by wiping from left to right horizontally.

$\begin{array}{ccc}\text { Correct } & \text { Incorrect } & \text { Missing } \\ (\mathrm{n}, \%) & (\mathrm{n}, \%) & (\mathrm{n}, \%)\end{array}$

$519(94.3)$

$29(5.3)$

$2(0.4)$

$506(92.0)$

$44(8.0)$

543 (98.7)

7 (1.3)

$0(0.0)$

$490(89.1)$

59 (10.7)

$1(0.2)$

$392(71.3)$

155 (28.2)

$3(0.5)$

313 (56.9)

$235(42.7)$

$2(0.4)$

478 (86.9)

70 (12.7)

$2(0.4)$

313 (56.9)

$235(42.7)$

$2(0.4)$

465 (84.5)

83 (15.1)

$466(84.8)$

81 (14.7)

$480(87.3)$

$69(12.5)$

Mean \pm standard deviation

$9.7 \pm 1.5$ 


\section{Adherence to safe injection practice guidelines}

The results of adherence to safe injection practice guidelines were as shown in Table 3. Of the 550 respondents, 384 (69.8\%) answered that they do not follow the guideline "Syringes and injection needles that were used by patients at home are collected by the institution"; $272(49.5 \%)$ answered that they do not follow the guideline "Syringes and injection needles are opened in the patients' vicinity."; $238(43.3 \%)$ answered that they do not follow the guideline "When opening an injectable drug that can be used for a long term, the disposal date must be written on the bottle". Finally, 7 respondents (1.3\%) answered that "Once injection needles are replaced, the syringe can be reused" is correct. In general, respondents followed 17-18 guideline practices out of the 24-guideline practices outlined.

Table 3. Adherence to guideline of safe injection practice

$(n=550)$

\begin{tabular}{|c|c|c|c|}
\hline & How much have you performed the following for the last one year? & $\begin{array}{l}\text { Non-compliant } \\
(\mathrm{n}, \%)\end{array}$ & $\begin{array}{c}\text { Compliant } \\
(\mathrm{n}, \%)\end{array}$ \\
\hline 1. & Syringes and injection needles that were used by patients at home are collected by the institution. & $384(69.8)$ & $166(30.2)$ \\
\hline 2. & Syringes and injection needles are opened in the patients' vicinity. & $272(49.5)$ & $278(50.5)$ \\
\hline 3. & When opening an injectable drug that can be used long term, the disposal date must be written on the bottle. & $238(43.3)$ & $312(56.7)$ \\
\hline 4. & A fluid set is replaced with a new one every 48 hours. & $192(34.9)$ & $358(65.1)$ \\
\hline 5. & When opening an injectable drug, the expiration date must be confirmed. & $191(34.7)$ & $359(65.3)$ \\
\hline 6. & When total parenteral nutrition (TPN) is given, a fluid set is replaced with a new one every 24 hours. & $188(34.2)$ & $362(65.8)$ \\
\hline 7. & When opening an injectable drug that can be used long term, the opening date must be written on the bottle. & $176(32.0)$ & $374(68.0)$ \\
\hline 8. & The injection area is replaced with a new one every $48-72$ hours. & $161(29.3)$ & $389(70.7)$ \\
\hline 9. & When inserting a peripheral vein catheter, the insertion date must be written. & $160(29.1)$ & $390(70.9)$ \\
\hline 10. & Blood, blood products, or lipid drugs are replaced with new ones as well as a fluid set whenever a new bottle is used. & $160(29.1)$ & $390(70.9)$ \\
\hline 11. & When preparing for injection, the drug name and dose in the injection bottle must be read and confirmed. & $158(28.7)$ & $392(71.3)$ \\
\hline 12. & When injecting a different drug in the identical patient, a new injection needle and syringe were used. & $149(27.1)$ & $401(72.9)$ \\
\hline 13. & One or more patients were injected with one bottle of injectable drug. & $146(26.5)$ & $404(73.5)$ \\
\hline 14. & In an emergency case, both previously opened injectable drugs and syringes are discarded. & $143(26.0)$ & $407(74.0)$ \\
\hline 15. & Whether there is flare or pain in the peripheral vein catheter insertion area is confirmed. & $129(23.5)$ & $421(76.5)$ \\
\hline 16. & Whenever injecting drugs for multiple use to multiple patients, new needles and syringes were used. & $127(23.1)$ & $423(76.9)$ \\
\hline 17. & You used an opened injectable drug that you did not open yourself. & $127(23.1)$ & $423(76.9)$ \\
\hline 18. & Injection was done without disinfecting the rubber plug of a vial. & $109(19.8)$ & $441(80.2)$ \\
\hline 19. & Previously used syringes/injection needles were placed with new syringes/injection needles in the same tray. & $102(18.5)$ & $448(81.5)$ \\
\hline 20. & Regardless of your intention, you have reused an injectable drug. & $98(17.8)$ & $452(82.2)$ \\
\hline 21. & You have disinfected a syringe or an injection needle. & $96(17.5)$ & $454(82.5)$ \\
\hline 22. & Injectable drugs that remained after use with one patient were placed at one location to use them for other patients. & $96(17.5)$ & $454(82.5)$ \\
\hline 23. & For reuse of vials for multiple use, injection needles, cannulas etc., were put in the rubber part of vials. & $91(16.5)$ & $459(83.5)$ \\
\hline 24. & For doing tasks quickly, you have put vials for multiple use in your uniform pocket. & $83(15.1)$ & 467 (84.9) \\
\hline & Mean \pm standard deviation & \multicolumn{2}{|c|}{$17.13 \pm 7.26$} \\
\hline
\end{tabular}




\section{Original Articles}

\section{Differences among adherences to safe injection practice guidelines}

The results of the univariate analyses showing the differences in adherence to safe injection practices were as provided in Table 4. Institution type $(p<.001)$, occupation type $(p<.001)$, work experience $(p<.05)$, training experience $(p<.001)$, guidelines/regulations on drug storage and management $(p<.001)$, patient safety culture $(p<.001)$ differed significantly between the compliant and non-compliant group.

Table 4. Differences between guideline compliant and non-compliant group

\begin{tabular}{|c|c|c|c|c|}
\hline & \multirow{2}{*}{ Variable } & \multicolumn{2}{|c|}{ Adherence $(\mathrm{n}, \%)^{1)}$} & \multirow{2}{*}{$x^{2}(p$-value $)$} \\
\hline & & Complaint & Non-compliant & \\
\hline \multirow[t]{2}{*}{ Institution type } & Tertiary or general hospital & $184(60.7)$ & $119(39.3)$ & $12.38(p<.000)$ \\
\hline & $\begin{array}{l}\text { Clinics, specialized hospital, nursing home, } \\
\text { public health center }\end{array}$ & $185(74.9)$ & $62(25.1)$ & \\
\hline \multirow[t]{3}{*}{ Type of occupation } & Physician & $44(66.7)$ & $22(33.3)$ & $42.85(p<.000)$ \\
\hline & Nurse & $207(80.2)$ & $51(19.8)$ & \\
\hline & Nursing assistant and others & $118(52.2)$ & $108(47.8)$ & \\
\hline \multirow[t]{2}{*}{ Work experience } & 5 years or less & $173(61.6)$ & $108(38.4)$ & $7.94(.005)$ \\
\hline & More than 5 years & $196(72.9)$ & $73(27.1)$ & \\
\hline \multirow{2}{*}{$\begin{array}{l}\text { Training experience } \\
\text { (for the last one year) }\end{array}$} & Yes & $265(74.6)$ & $90(25.4)$ & $23.12(p<.000)$ \\
\hline & No & $104(54.5)$ & $87(45.5)$ & \\
\hline \multirow{2}{*}{$\begin{array}{l}\text { Awareness of safe injection } \\
\text { practice }\end{array}$} & Greater than mean & $240(71.6)$ & $95(28.4)$ & $8.04(.005)$ \\
\hline & Less than mean & $129(60.0)$ & $86(40.0)$ & \\
\hline \multirow{2}{*}{$\begin{array}{l}\text { Guidelines/regulations } \\
\text { on drug storage and } \\
\text { management }\end{array}$} & Yes & $349(69.8)$ & $151(30.2)$ & $13.32(p<.000)$ \\
\hline & No & $20(43.5)$ & $26(56.5)$ & \\
\hline \multirow[t]{2}{*}{ Patient safety culture } & Greater than mean & $209(75.7)$ & $67(24.3)$ & $19.87(p<.000)$ \\
\hline & Less than mean & $151(57.6)$ & $111(42.4)$ & \\
\hline
\end{tabular}

1) The total is not 550 in some cases due to missing variables 
5. Factors related to adherence to safe injection practice guidelines: results of multivariate logistic regression

Results showed that safe injection practices were influenced by the status of training experience related to safe injection practices, patient safety culture, and type of occupation. Multivariate logistic regression identified three factors in those who were more likely to adhere to safe injection practice guidelines: (1) having training experience about patient safety in the last one year (OR, 2.24; 95\% CI, 1.47, 3.40). (2) Having a higher patient safety culture score (OR 2.12; 95\% CI, 1.43, 3.14). (3) Being a physician (OR 2.23; 95\% CI, 1.18, 4.22) or a nurse (OR, 4.17; 95\% CI, 2.32, 7.50) compared to being nursing assistants and other professionals. The factors - institution type, having a work experience of more than 5 years, having awareness of safe injection practices, and having guidelines or regulations on drug storage and management in working institutions were found insignificant (Table 5). In step 3, Cox \& Snell $\mathrm{R}^{2}$ was found to be 0.135 and Nagelkerke $\mathrm{R}^{2}$ was 0.188 . The result of Hosmer and Lemeshow test was 0.903 .

Table 5. Influencing factors in adherence to guideline of safe injection practice

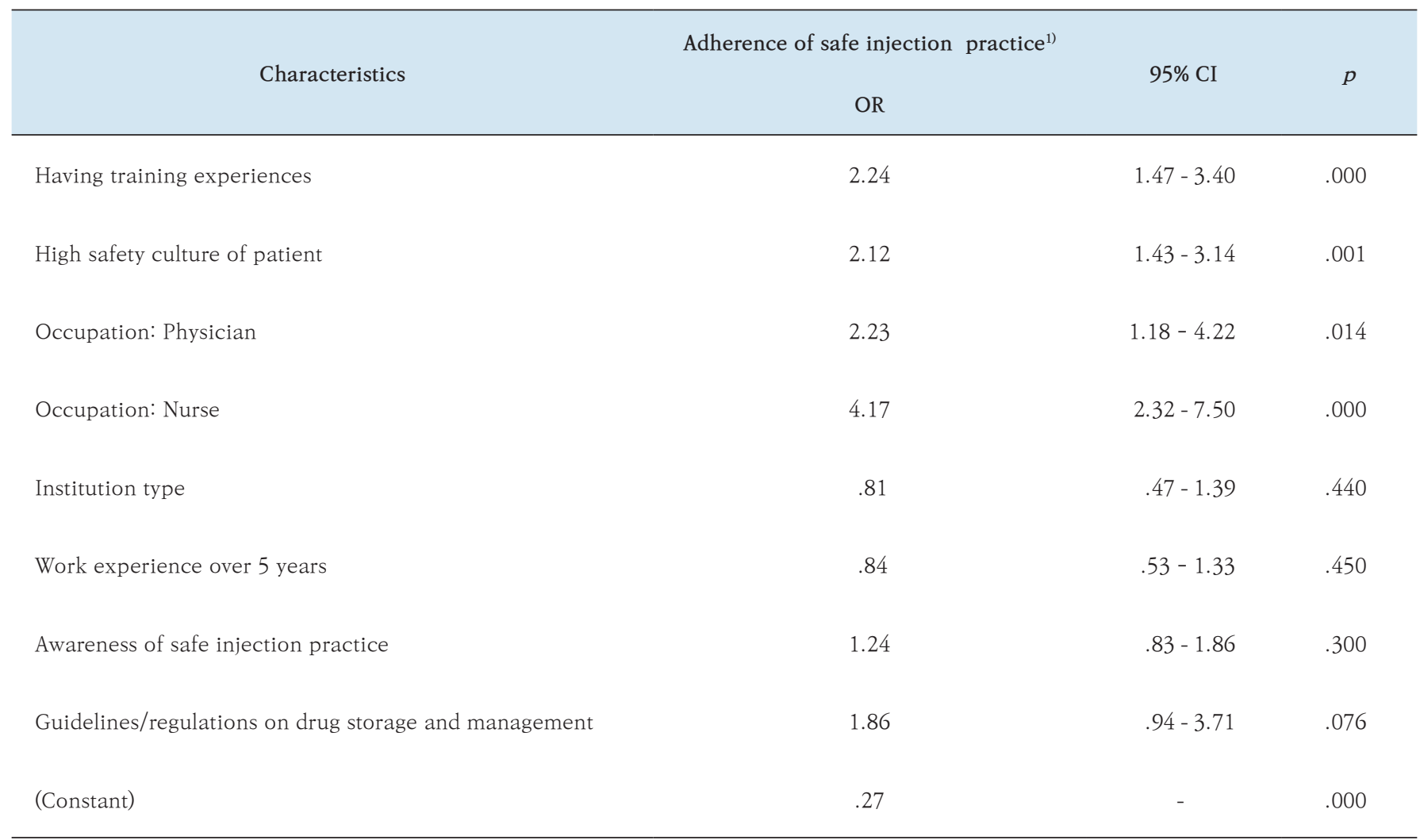

OR odds ratio; 95\% CI confidence interval

1) The dependent variable had two categories: Greater and lower than mean of injection practice adherence 


\section{Original Articles}

\section{Barriers to adherence to safe injection practice guidelines}

Barriers to adherence to safe injection practice guidelines were as shown in Table 6. Of the 550 respondents, $244(44.4 \%)$ answered that "It is unfortunate to discard injectable drugs only after using some"; 196 (35.6\%) answered "It is cumbersome to comply with sterilization method at every injection"; and 195 (35.5\%) answered "There is no enough space to store new drugs or products"; $178(32.4 \%)$ and $154(28.0 \%)$ answered that stored drugs and stored products, including syringes, needles, fluid sets, were insufficient, respectively; $153(27.8 \%)$ answered that there was not enough opportunity to learn about sterilization methods; $148(26.9 \%)$ answered that there is no culture that promotes adherence to sterilization methods; and 147 (26.7\%) answered that there is not enough time to practice sterilization methods.

Table 6. Barriers to adherence of safe injection guideline

$(n=550)$

$\begin{array}{lcc}\text { Question } & \text { Yes } & \text { No } \\ & (\mathrm{n}, \%) & (\mathrm{n}, \%)\end{array}$

1. It is unfortunate to discard injectable drugs only after using some.

$244(44.4) \quad 304(55.3)$

2. It is cumbersome to comply with sterilization methods at every injection.

$196(35.6) \quad 354(64.4)$

3. There is not enough space to store new drugs or products.

$195(35.5) \quad 355(64.5)$

4. The amount of stored drugs is insufficient.

$178(32.4) \quad 371(67.5)$

5. The amount of products such as stored syringes, injection needles, fluid sets etc., are insufficient.

6. There is not enough opportunity to learn about sterilization methods.

7. There is no atmosphere of adherence to sterilization methods.

8. There is not enough time to practice sterilization methods.

9. The contents of the sterilization methods specified in the guidelines are confusing or not accurate.

10. Caution is always taken to prevent the amount of injection-related waste products from becoming excessive.

11. After acquiring a license, it is easy to forget about the sterilization methods because of a lack of repeated education or training.

12. No payment is made for the remaining drugs after use.

13. The cost of drugs is too expensive.

14. There are no disadvantages or consequences even if one does not adhere to the sterilization methods.

* The total is not 550 in some cases due to missing variables 


\section{Discussion}

The objective of this study was to assess the awareness regarding safe injection practices, adherence to guidelines for safe injection practices for healthcare providers, and barriers in promotion of patient safety. Awareness regarding safe injection practices scored an average of 9.8 out of 12 points. The most number of incorrect answers were to the questions related to alcohol-soaked cotton and adequate disinfecting time. 10\% and $15 \%$ of the respondents gave incorrect answers to questions related to confirmation about multiple patient use and usage of large amounts of solvent fluid to resolve injection, respectively. In addition, "Once injection needles are replaced, syringes can be reused" was $1.3 \%$, so there has been a concurrent risk of reusing syringes even though outbreaks recently occurred in Korea.

In terms of adherence to guidelines for safe injection practices, the highest number of respondents who did not adhere to safety regulations was seen in the responses to "Syringes and injection needles that were used by patients at home are collected by the institution." With respect to the increase in chronic diseases that require self-injections, including diabetes mellitus and rheumatoid arthritis, it is necessary to consider developing a policy for prevention of secondary infection. Sudesh Gyawali et al. emphasized the need for provision of safety boxes for the disposal of used syringes and a continuous and repeated training process for medical staff on waste product management [18]. Further, 30\% of all the respondents answered that they did not note the opening date/disposal date or did not confirm the expiration date when opening injectable drugs. About 20\% reported cases related to the reuse of injection-related products, such as opening syringes or injection needles in advance, not using new needles and syringes for multipleuse medication injections, using injectable drugs that they themselves had not opened, or placing previously used syringes/injection needles on a tray. Therefore, it was confirmed that the safety precautions of healthcare providers regarding injection safety were not adequate.

Results showed that safe injection practices were affected by status of training related to injection safety practices for the previous year, patient safety culture, and type of occupation. The probability of practicing safe injection-related activities was more than twice greater in the group that underwent training related to injection safety practices than the group that did not undergo training; the same was true for the physician and nurse groups. With regard to patient safety culture, positive workplace and organizational culture was significantly associated with medication errors and hospital acquired infection [19]. Several studies have attempted to find the relationship between safety culture and safety behavior, though these have tended to focus more on hand hygiene [25]. The disconnection between intent and action appears to be influenced by organizational structure as well as cultural beliefs [20]. Marzieh Javadi et al. mentioned that normative beliefs and control beliefs related to safety culture also have to be improved to inspire the provider's intention to implement patient safety behavior simultaneously [21-22]. Organizational structure [20] and social cohesiveness [24] of among team members also 
influence the success of interventions for improving compliance.

With respect to occupation, respondents who were neither doctors nor nurses were thought to have lacked the opportunity to receive training on safe injection practices and to have a sufficient recognition of patient safety culture. A study reported the negative relationship between the number of hours per resident day of licensed practical nurses and food enjoyment in nursing homes, but relatively few studies have been devoted to differences in adherence and barriers to safe practices by type of occupation.

Further research is required to determine these factors comprehensively. Cresswell and Monrouxe found that awareness varied across the medical junior doctors and medical students [23]. There was a strong belief that evidence for the effectiveness of good hygiene behaviors is lacking, and doctors and medical students had a predominant view that hygiene was an additional rather than integral part of the process. The authors emphasized ownership of patient safety behavior and understanding the doctor's role in developing the hygiene culture. The Speaking Up for Safety Program focused on how leadership support, HCW awareness, supply availability, and culture change increased compliance to hand hygiene [24].

Regarding barriers to medication injections, $44.4 \%$ of the respondents answered with "It is unfortunate to have to discard injectable drugs without using them entirely". To resolve this, it is necessary to have a close consultation between insurance companies and manufacturing companies. The Korean health insurance system is a unique hybrid single payer system where all people residing in South Korea are eligible for coverage under the National Health Insurance Program. Regarding injectable drugs whose unit prices are determined by packaging unit, the current insurance payment structure leaves some room for ambiguity regarding the reuse of drugs in cases where some of the injectable drugs are left over after use [25]. Manufacturing companies need to make product units with smaller amounts; also, subdividing insurance payments into units with smaller amounts will help lower the risk of reuse. Most respondents also found it cumbersome to comply with the sterilization procedures for every injection; the improvement of recognition using continuous and repeated education or modification of existing safety processes or campaigns can make patient safety culture among the providers the most effective method of counteracting this [26].

Approximately $80 \%$ of the respondents confirmed that their institutions had guidelines and regulations on drug storage and management. However, approximately 50\% of them responded that they had not undergone any training related to injection safety held by their institutions or external agencies, which indicates a lack of a continuous training process on injection practices. Also, regarding barriers safe injection practices, the high number of responses on "After acquiring a license, it is easy to forget about sterilization methods because of a lack of repeated education or training" and "There are no enough opportunities to learn about sterilization methods" suggests the importance of systematic and continual training in each area. Kossover-Smith et al. emphasized that training in basic prevention must be repeated continuous and repeated process right from the 
early years of service of public healthcare service providers [27].

Naveed Zafar Janjua et al. argued that the introduction of syringes combined with reuse prevention devices should be considered at the national level [28]. Insufficient injectable medicine and related products and no reimbursements for the remaining drug after use were mentioned as barriers in adherence to safe injection guidelines. This means that the government should make reuse-prevention syringes available at lower prices and that it should gradually phase out the sale of the existing syringes and incentivize manufacturers that make reuse-prevention injections. However, to achieve this, close consultation among the government, hospitals, and manufacturers is required, and there is a limit to mandating the exclusive use of reuse-prevention syringes.

This study had several limitations. Participants (healthcare providers) were selected as a convenience sample and both online and offline methods; thus, the findings may not be entirely representative of healthcare providers in Korea. Second, despite asking the participants if they were working on medication injection or were managing and supervising medication injections the last one year to screen respondents, the job on medication injection including storage, preparation, and administration was not specified. It might vary among healthcare providers and would be useful to know more about the specific job related to injection and awareness level. Third, the measure for adherence to guidelines was based on selfreport; thus, there might be the possibility of social desirability bias. Nevertheless, this study revealed factors associated with adherence to guidelines in healthcare providers. Further study could be conducted to identify effective strategies for prevention of outbreak

\section{Conclusions}

This study's results indicate that controllable factors like training experience of healthcare providers and patient safety culture were positively associated with adherence to safe injection practices. The study concluded that healthcare providers be continually and repeatedly trained in safe injection practices to promote patient safety. Additionally, there should be an increased focus on developing and implementing policies to improve patient safety.

\section{Acknowledgements}

This work was supported by a research program funded by the National Evidence-Based Healthcare Collaborating Agency (NECA) in Korea (NH16-004).

\section{References}

1. Arnold S, Melville SK, Morehead B, Vaughan G, Moorman A, Crist MB. Notes from the Field: Hepatitis C Transmission from Inappropriate $\mathrm{Re}^{-}$ use of Saline Flush Syringes for Multiple Patients in an Acute Care General Hospital - Texas, 2015. Mmwr-Morbidity and Mortality Weekly Report. 2017;66(9):258-60.

2. Kim IH. Recent outbreaks of hepatitis $\mathrm{C}$ virus infection in Korea and strategy for prevention. Journal of Korean Medical Association. 2016;59(12):91215. 
3. Fabrizi F, Dixit V, Messa P, Martin P. Transmission of hepatitis B virus in dialysis units: a systematic review of reports on outbreaks. International Journal of Artificial Organs. 2015;38(1):1-7.

4. Fabrizi F, Messa P. Transmission of hepatitis $\mathrm{C}$ virus in dialysis units: a systematic review of reports on outbreaks. International Journal of Artificial Organs. 2015;38(9):471-80.

5. Nguyen DB, Gutowski J, Ghiselli M, Cheng T, Bel Hamdounia S, Suryaprasad A, et al. A large outbreak of hepatitis $C$ virus infections in a hemodialysis clinic. Infection Control and Hospital Epidemiology. 2016;37(2):125-33.

6. Centers for Disease Control and Prevention. Healthcare-Associated Hepatitis B and C Outbreaks Reported to the Centers for Disease Control and Prevention (CDC) 2008-2017. 2018 [last updated 2018 Sep 18]. Available from: https://www. cdc.gov/hepatitis/Outbreaks/HealthcareHepOutbreakTable.htm.

7. Kim HJ, Cho Y, Lee S, Kook Y, Lee D, Lee J, et al. Mycobacterium massiliense outbreak after intramuscular injection, South Korea. Epidemiology and Infection. 2012;140(10):1880-7.

8. Jung SY, Kim BG, Kwon D, Park JH, Youn SK, Jeon $S$, et al. An outbreak of joint and cutaneous infections caused by non-tuberculous mycobacteria after corticosteroid injection. International Journal of Infectious Diseases. 2015;36(C): 62-9.

9. Manchikanti L, Falco FJ, Benyamin RM, Caraway DL, Helm Ii S, Wargo BW, et al. Assessment of infection control practices for interventional techniques: a best evidence synthesis of safe injection practices and use of single-dose medication vials. Pain Physician. 2012;15(5):E573-614.

10. Dolan SA, Arias KM, Felizardo G, Barnes S, Kras- ka S, Patrick M, et al. APIC position paper: Safe injection, infusion, and medication vial practices in health care. American Journal of Infection Control. 2016;44(7):750-7.

11. WHO Guidelines Approved by the Guidelines Review Committee. WHO guideline on the use of safety-engineered syringes for intramuscular, intradermal and subcutaneous injections in health care settings. Geneva, Switzerland: World Health Organizaiton; 2016. p. 49.

12. Pugliese G, Gosnell C, Bartley JM, Robinson S. Injection practices among clinicians in United States health care settings. American Journal of Infection Control. 2010;38(10):789-98.

13. Gounder P, Beers R, Bornschlegel K, Hinterland $\mathrm{K}$, Balter S. Medication injection safety knowledge and practices among anesthesiologists: New York State, 2011. Journal of Clinical Anesthesia. 2013; 25(7):521-8.

14. Lunden A, Teräs M, Kvist T, Häggman-Laitila A. A systematic review of factors influencing knowledge management and the nurse leaders' role. Journal of Nursing Management. 2017;25(6):407-20.

15. Olsson C, Forsberg A, Bjerså K. Safety climate and readiness for implementation of evidence and person centered practice - A national study of registered nurses in general surgical care at Swedish university hospitals. BMC Nursing. 2016;15(1):54.

16. Chung YS, Choi JY, Han MG, Park KR, Park SJ, Lee $\mathrm{H}$, et al. A large healthcare-associated outbreak of hepatitis $\mathrm{C}$ virus genotype $1 \mathrm{a}$ in a clinic in Korea. Journal of Clinical Virology. 2018;106:53-7.

17. Kim JE, Kang MA, An KA, Sung YH. A survey of nurses' perception of patient safety related to hospital culture and reports of medical errors Journal of Korean Clinical Nursing Research. 
2007;13(3):169-79.

18. Gyawali S, Rathore DS, Kc B, Shankar PR. Study of status of safe injection practice and knowledge regarding injection safety among primary health care workers in Baglung district, western Nepal. BMC International Health and Human Rights. 2013;13: 3 .

19. Braithwaite J, Herkes J, Ludlow K, Testa L, Lamprell G. Association between organisational and workplace cultures, and patient outcomes: systematic review. BMJ Open. 2017;7(11):e017708.

20. Butenko S, Lockwood C, McArthur A. Patient experiences of partnering with healthcare professionals for hand hygiene compliance: a systematic review. JBI Database of System Reviews and ImpleMateria socio-medica. 2017;15(6):1645-70.

21. Javadi M, Kadkhodaee M, Yaghoubi M, Maroufi M, Shams A. Applying theory of planned behavior in predicting of patient safety behaviors of nurses. Materia socio-medica. 2013;25(1):52-5.

22. Wakefield JG, McLaws ML, Whitby M, Patton L. Patient safety culture: factors that influence clinician involvement in patient safety behaviours. Quality and Safety in Health Care. 2010;19(6):585-91.

23. Cresswell P, Monrouxe LV. 'And you'll suddenly realise 'I've not washed my hands': medical students', junior doctors' and medical educators' narratives of hygiene behaviours. BMJ Open. 2018; 8(3): e018156.

24. Linam WM, Honeycutt MD, Gilliam CH, Wisdom CM, Deshpande JK. Impact of a successful speaking up program on health-care worker hand hygiene behavior. Pediatric Quality and Safety. 2017;2(4): e035.

25. Health Insurance Review \& Assessment Service. Korean National Health Insurance Fee Schedule.
Gangwon-do, Korea: Health Insurance Review \& Assessment Service; 2017.

26. Ismail AA, Mahfouz MS, Makeen A. Injection safety among primary health care workers in Jazan Region, Saudi Arabia. International Journal of Occupational and Environmental Medicine. 2014;5(3): 155-63.

27. Kossover-Smith RA, Coutts K, Hatfield KM, Cochran R, Akselrod H, Schaefer MK, et al. One needle, one syringe, only one time? A survey of physician and nurse knowledge, attitudes, and practices around injection safety. American Journal of Infection Control. 2017;45(9):1018-23.

28. Janjua NZ, Butt ZA, Mahmood B, Altaf A. Towards safe injection practices for prevention of hepatitis C transmission in South Asia: challenges and progress. World Journal Gastroenterology. 2016;22(25):5837-52. 